Pacific Journal of Mathematic 


\title{
UNIFORM SUBGROUPS AND ERGODIC ACTIONS OF EXPONENTIAL LIE GROUPS
}

\section{ROBERT J. ZIMMER}

\begin{abstract}
We show that the restriction of an ergodic action of an exponential solvable Lie group to a uniform subgroup is still ergodic provided that the restriction to the commutator subgroup is ergodic. This complements similar results previously obtained for semi-simple and nilpotent Lie groups.
\end{abstract}

1. Introduction. Suppose $G$ is a locally compact group which acts ergodically on a standard Borel space with quasi-invariant measure $(S, \mu)$, and that $H$ is a closed subgroup of $G$. An important problem in ergodic theory is to determine when the restriction of the $G$-action to $H$ is still ergodic. For certain $G$ and $H$ answers are known completely. For example, let $G$ be a noncompact connected simple Lie group with finite center and $H=\Gamma$ a lattice subgroup in $G$. If $S$ is a transitive $G$-space, then C. C. Moore [6] has shown that $\Gamma$ will be ergodic on $S$ if and only if the stability groups of the $G$-action are not compact. If $S$ is properly ergodic; i.e., ergodic but not transitive (modulo null sets), then the author has shown in [9] that in all such cases $\Gamma$ is ergodic on $S$. If $G$ is a connected, simply connected, nilpotent Lie group, we also showed in [9], based on results in [8], that the restriction of an ergodic $G$-action to an arbitrary lattice subgroup $\Gamma$ is ergodic provided that the restriction to $[G, G]$ is ergodic. The point of this paper is to provide a similar result for exponential solvable Lie groups. By a uniform subgroup of $G$ we mean one whose quotient is compact with a finite $G$-invariant measure. Our main result is the following.

THEOREM 1. Suppose $G$ is a connected, simply connected, exponential solvable Lie group. Let $S$ be an ergodic G-space. If $[G, G]$ is also ergodic on $S$, then $\Gamma$ is ergodic on $S$ for every uniform subgroup $\Gamma \subset G$.

In proving the results in [9] stated above concerning simple and nilpotent Lie groups, we made important use of certain properties of the space of orbits of unitary representations of such groups. Results of this type will be equally important for us in the present situation and these will follow from recent work of $R$. Howe and C. C. Moore [5].

2. Projective kernels. In this section we present some results 
on projective kernels of unitary representations that we will need to prove Theorem 1 . We suppose $G$ is a locally compact second countable group. By a representation of $G$ we shall mean a strongly continuous unitary representation of $G$ on a separable Hilbert space. The set of equivalence classes of irreducible representations will as usual be denoted by $\hat{G}$. If $M \subset G$ is a closed subgroup and $\sigma$ is a representation of $M$, we will denote the representation of $G$ induced from $\sigma$ by $\operatorname{ind}_{M}^{F}(\sigma)$. The kernel of a representation $\pi$ is the set of elements with $\pi(g)=I$ and the projective kernel of $\pi$, which we denote by $P_{\pi}$, is $\{g \mid \pi(g) \in C I\}$. The significance of $P_{\pi}$ for studying the asymptotic behavior of $\pi$ is demonstrated in [5]. Our first aim here is to show that for exponential solvable Lie groups, $P_{\pi}$ cannot be too large for suitable $\pi$.

We recall that a solvable Lie group is exponential if the exponential map from the Lie algebra into the group is a surjection. For example, connected nilpotent Lie groups are exponential. Two nonnilpotent exponential groups that arise in the study of the structure of a general exponential group are the following. Let $S_{1}$ be the $a x+b$ group, i.e., $S_{1}$ is a semi-direct product $A$ (S) $B$, where $A$ is the multiplicative group of reals, $B$ is the additive group of reals, and $A$ acts by automorphisms on $B$ by multiplication. We define $S_{2}$ to be the semi-direct product $\boldsymbol{R}(S)$ where the action of $\boldsymbol{R}$ by automorphisms of $\boldsymbol{C}$ is given by $t \cdot z=[\exp (1-i \sigma) t] z$, where $\sigma$ is a nonzero real number. The important role played by $S_{1}$ and $S_{2}$ in the structure of a general exponential solvable group can be seen from [1, Theorem VII. 2.2] or [2, Lemma 2.3] for example.

Proposition 2. Let $G$ be a connected, simply connected, exponential solvable Lie group, and suppose that $\pi$ is an infinite dimensional irreducible unitary representation. Let $H \subset G$ be the closure of the subgroup $P_{\pi}[G, G]$. Then $G / H$ is not compact.

Proof. We argue by induction on the dimension of $G$. We note first that if $\pi=\operatorname{ind}_{G_{0}}^{G}(\sigma)$ where $G_{0}$ is a connected normal subgroup, then $P_{\pi} \subset G_{0}$. It follows that the theorem is true for $G$ nilpotent, since $\pi$ is induced by a representation of a connected subgroup of $G$ containing $[G, G]$. (See [1, Chapter V].) Thus, we suppose $G$ is exponential, nonnilpotent, and that the theorem is true for all connected simply connected exponential Lie groups of strictly lower dimension. Let $N$ be the nilradical of $G$. If $P_{\pi} \subset N$, we are clearly done since $[G, G] \subset N$ as well. If not, choose $X \in L(G)$, the Lie algebra of $G$, such that $X \notin L(N)$ and $\exp (X) \in P_{\pi}$. We note that $X$ must have some nonzero eigenvalue under the adjoint representation [4, p. 131]. By a result of Auslander and Green [2, Lemma 
2.3], there is a subgroup $S \subset G$ isomorphic to either $S_{1}$ or $S_{2}$, and such that the Lie algebra $L(S)$ satisfies $X \in L(S) \subset L(G)$ with $X$ a regular element in $L(S)$. By the Mautner phenomenon for $S_{i}$ (see [1, Chapter II], for example), $\exp (X) \in P_{\pi}$ implies that $\pi$ is the identity on $[S, S]$. Let (ker $\pi)_{0}$ be the connected component of the identity in $\operatorname{ker}(\pi)$. It follows that $G_{1}=(\operatorname{ker} \pi)_{0} \cap[G, G]$ is a connected normal subgroup of nonzero dimension. The representation $\pi$ then factors to a representation $\tilde{\pi}$ of $\widetilde{G}=G / G_{1}$, which is also connected, simply connected, and exponential. Since we have a continuous surjection $G \overline{P_{\pi}[G, G]} \rightarrow \widetilde{G} / \overline{P_{\widetilde{\pi}}[\widetilde{G}, \widetilde{G}]}$, the rasult follows by the inductive hypothesis.

We remark that Proposition 2 is not true for connected simply connected solvable Lie groups in general, as one can easily see by examining the representations of the universal covering group of the group of (orientation preserving) rigid motions of the plane.

We now make some observations of a somewhat different type concerning projective kernels. Suppose $\pi \in \hat{G}$ is represented on a Hilbert space $\mathscr{H}(\pi)$, and let $B$ and $X$ be the vectors of norm 1 and the projective space of $\mathscr{C}(\pi)$ respectively. Then $\pi$ defines an action of $G$ on $B$ and $X$. An action of a locally compact group on a standard Borel space will be called smooth if every ergodic (quasiinvariant) probability measure is supported on an orbit. This is known to be equivalent to a large number of other regularity conditions [3]. The hypothesis of the following result is clearly inspired by the results of Howe and Moore [5].

Proposition 3. Suppose $\pi$ is a representation of a locally compact group $G$ and that for each $v, w \in \mathscr{H}(\pi),\langle\pi(g) v \mid w\rangle \rightarrow 0$ as $g \rightarrow \infty$ modulo $G / P_{\pi}$. If $\pi\left(P_{\pi}\right)$ is closed, then the actions of $G$ on $B$ and $X$ are smooth.

Proof. As observed in [9, Proposition 2.2], it suffices to show that the action on $B$ is smooth. To do this, it suffices to show that the orbits in $B$ are norm closed [3]. Let $v \in B, g_{n} \in G$, and suppose $v \cdot g_{n} \rightarrow w \in B$. Then $g_{n}$ must be bounded when projected to $G / P_{\pi}$. There exists a section $G / P_{\pi} \rightarrow G$ that is bounded on compact sets, and so there exist $k_{n} \in G,\left\{k_{n}\right\}$ bounded, such that $g_{n} k_{n} \in P_{\pi}$. We can suppose $k_{n} \rightarrow k$, so $v \cdot g_{n} k_{n} \rightarrow w \cdot k$. Since $g_{n} k_{n}$ acts by scalars and since $\pi\left(P_{\pi}\right)$ is closed, there exists $y \in P_{\pi}$ such that $v \cdot y=\lim v \cdot g_{n} k_{n}$. Thus $v \cdot y=w \cdot k$ and $w$ is in the orbit of $v$.

We will want to apply Proposition 3 to exponential groups, and hence need the following result.

Proposition 4. If $\pi \in \hat{G}$ where $G$ is a connected simply con- 
nected exponential solvable Lie group, then $\pi\left(P_{\pi}\right)$ is closed.

Proof. If $\pi$ is one-dimensional, this is clear, so we need consider only the case in which $\pi$ is infinite dimensional. We can clearly reduce readily to the case in which $K=\operatorname{ker}(\pi)$ is discrete. Using the existence of subgroups isomorphic to $S_{i}$ and the Mautner phenomenon as in Proposition 2, we see that we must then have $P_{\pi} \subset N$, where $N$ is the nilradical of $G$. Furthermore, $P_{\pi} \subset Z(G)$, the center of $G$. This follows from the fact that if $\varphi: G \rightarrow G / K$ is the natural projection, then $\varphi^{-1}(Z(G / K))=Z(G)$ since $G$ is connected and $K$ is discrete, and the observation that $\varphi\left(P_{\pi}\right) \subset Z(G / K)$. Thus, $P_{\pi} \subset(N \cap Z(G)) \subset Z(N)$. By Mackey's analysis, we can represent $\pi$ by $\pi=\operatorname{ind}_{M}^{G}(\sigma)$ where $M$ is a subgroup with $N \subset M \subset G, \sigma \in \hat{M}$, and $\sigma \mid N$ is a multiple of a representation $\alpha \in \hat{N}$. Since $\alpha$ is irreducible, we have that $Z(N) \subset P_{\alpha}$. By the subgroup theorem, we can write $\pi \mid N=n \int_{G / M}^{\oplus} \alpha \cdot g$, where $n \in\{\infty, 1,2, \cdots\}$ and $\alpha \cdot g$ refers to the action of $G$ on $\hat{N}$. From this we see that for $x \in Z(N)$, we have $x \in P_{\pi}$ if and only if $\alpha\left(g x g^{-1}\right)=\alpha(x)$ for all $g \in G$, and in that case $\pi(x)=\alpha(x)$ considered as scalars. For each $g$, let $\varphi_{g}$ be the character of $Z(N)$ defined by $\varphi_{g}(x)=\alpha\left(g x g^{-1}\right)$. Identifying $Z(N)$ as a vector group, $\left\{\varphi_{g} \mid g \in G\right\}$ is then a connected subset of the dual vector space $Z(N)^{*}$. Therefore, we have $x \in P_{\pi}$ if and only if $\left\{\varphi_{g}\right\}$ is contained in an affine space which is a translate of $\operatorname{ker} x \subset Z(N)^{*}$. From this it becomes clear that $\pi\left(P_{\pi}\right)=\alpha\left(P_{\pi}\right)$ is either $\{1\}$ or the entire unit circle.

3. Proof of Theorem 1. We now turn to the proof of Theorem 1. We recall that the definition of an ergodic $G$-space $(S, \mu)$ entails the presence of a jointly Borel $G$-action on $S$ under which $\mu$ is quasi-invariant and ergodic. We shall emply below some of the concepts and results from the cohomology theory of such actions, for which the reader is referred to [7], [9], and the bilbliography of these papers.

We let $N=[G, G]$ and suppose that the restriction of the $G$-action on $S$ to $N$ is ergodic. Suppose that for some uniform subgroup $\Gamma \subset G, \Gamma$ is not ergodic on $S$. By [9, Theorem 4.2], the product $G$-action on $S \times G / \Gamma$ is not ergodic. Since $\Gamma$ is uniform, we can write $\operatorname{ind}_{c}^{G}(I)=I \oplus \Sigma^{\oplus} \chi_{i} \oplus \Sigma^{\oplus} \pi_{i}$ where $\chi_{i}$ are nontrivial one-dimensional representations of $G$ and $\pi_{i}$ are infinite-dimensional irreducible representations. It follows from [9, Proposition 3.1] that for at least one $\chi_{i}$ or $\pi_{i}$, the restriction of the representation to a cocycle on $S \times G$ must contain the identity cocycle. If this were true for some $\chi_{i}$, it would then follow that $S \times G / \overline{\Gamma N}$ would not be ergodic, 
which is impossible since $S \times G / N$ is ergodic [9, Theorem 4.2]. Thus we can assume that some $\pi_{i}=\pi$ restricts to a cocycle on $S \times G$ which contains the identity. It follows from [9, Theorem 3.2] that (perhaps by passing to a $G$-invariant conull Borel subset of $S$ ) that there is then a $G$-map $\varphi: S \rightarrow X$, where $X$ is projective space in $\mathscr{H}(\pi)$. If $x \in X$, let $G_{x}$ be the stability group in $G$ at $x$. It follows from a result of Howe and Moore [5, Theorem 7.1] that $G_{x} / P_{\pi}$ is a compact group. From [5, Theorem 7.1] and Propositions 3 and 4, we know that $G$ acts smoothly on $X$, and so every orbit is homeomorphic to $G / G_{x}$ for some $x$ in the orbit, and is locally closed in $X$ [3]. Consider now the $H$-orbits in $X$ where $H=\overline{P_{\pi} N}$. For each $x$, $G_{x} H$ is closed in $G$. This follows from the fact that $G_{x} H$ is the inverse image under the natural projection to $G / P_{\pi}$ of $\left(G_{x} / P_{\pi}\right)\left(H / P_{n}\right)$ and the latter is closed since $G_{x} / P_{\pi}$ is compact. It follows that the $H$-orbit of $x \in X$ will be homeomorphic to the closed subset $H G_{x} / G_{x}$ of $G / G_{x}$, and will be a locally closed set in $X$. Since all $H$-orbits are locally closed, it follows that $H$ acts smoothly on $X$ [3]. But $\varphi_{*}(\mu)$ is $H$-quasi-invariant on $X$, and since $N$ is ergodic on $S, H$ clearly is as well, which implies that $\varphi_{*}(\mu)$ is ergodic under $H$ and by smoothness supported on an $H$-orbit $Y \subset X$. Let $S_{0}=\varphi^{-1}(Y)$, a conull Borel set in $S$. Then the set $\left\{(s, g) \mid s \in S_{0}, s g \in S_{0}\right\}$ is conull in $S \times G$. By Fubini's theorem, and since $\varphi$ is a $G$-map, we conclude that for almost all $y \in Y$, we have $y g \in Y$ for almost all $g$. Since $Y$ is $G$-homeomorphic to a closed subset of $G / G_{x}$ for some $x$, this implies that $y g \in Y$ for all $g \in G$. In other words, $Y$ contains a $G$-orbit, and since it is an $H$-orbit, we in fact have $Y \cong G / G_{x}$ for some $x$. But this implies $G_{x} H=G$, so that the map $G_{x} / P_{\pi} \rightarrow G / H$ is surjective. This is impossible since $G_{x} / P_{\pi}$ is compact while $G / H$ is not by Proposition 2. This contradiction completes the proof.

\section{REFERENCES}

1. L. Auslander, L. Green, and F. Hahn, Flows on homogeneous spaces, Annals of Math. Studies no. 53, Princeton, 1963.

2. L. Auslander and L. Green, G-induced flows, Amer. J. Math., 88 (1966), 43-60.

3. E. G. Effros, Transformation groups and $C^{*}$-algebras, Annals of Math., 81 (1965), 38-55.

4. G. Hochschild, The Structure of Lie Groups, Holden-Day, San Francisco, 1965.

5. R. Howe and C. C. Moore, Asymptotic properties of unitary representations, to appear, J. Functional Analysis.

6. C. C. Moore, Ergodicity of flows on homogeneous spaces, Amer. J. Math., 88 (1966), 154-178.

7. R. J. Zimmer, Extensions of ergodic group actions, Illinois J. Math., 20 (1976), $373-409$.

8. Compact nilmanifold extensions of ergodic actions, Trans. Amer. Math. Soc., 223 (1976), 397-406. 
9. R. J. Zimmer, Orbit spaces of unitary representations, ergodic theory, and simple Lie groups, Annals of Math., 106 (1977), 573-588.

Received February 21, 1978.

University of Chicago

CHICAGO, IL 60637 


\section{PACIFIC JOURNAL OF MATHEMATICS}

\section{EDITORS}

RICHARD ARENS (Managing Editor)

University of California

Los Angeles, California 90024

C. W. CurTis

University of Oregon

Eugene, OR 97403

C. C. MOORE

University of California

Berkeley, CA 94720
J. DUGUNDJI

Department of Mathematics University of Southern California Los Angeles, California 90007

R. FinN AND J. Milgram Stanford University Stanford, California 94305

\section{ASSOCIATE EDITORS}

E. F. BECKENBACH

B. H. NeumanN

F. WOLF

K. YoSHIDA

\section{SUPPORTING INSTITUTIONS}

UNIVERSITY OF BRITISH COLUMBIA CALIFORNIA INSTITUTE OF TECHNOLOGY UNIVERSITY OF CALIFORNIA MONTANA STATE UNIVERSITY UNIVERSITY OF NEVADA, RENO NEW MEXICO STATE UNIVERSITY OREGON STATE UNIVERSITY UNIVERSITY OF OREGON
UNIVERSITY OF SOUTHERN CALIFORNIA

STANFORD UNIVERSITY

UNIVERSITY OF HAWAII

UNIVERSITY OF TOKYO

UNIVERSITY OF UTAH

WASHINGTON STATE UNIVERSITY

UNIVERSITY OF WASHINGTON 


\section{Pacific Journal of Mathematics \\ Vol. 78, No. $1 \quad$ March, 1978}

Simeon M. Berman, A class of isotropic distributions in $\mathbf{R}^{n}$ and their

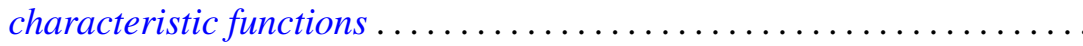

Ezra Brown and Charles John Parry, The 2-class group of biquadratic fields.

II ........................................ 11

Thomas E. Cecil and Patrick J. Ryan, Focal sets of submanifolds ....... 27

Joseph A. Cima and James Warren Roberts, Denting points in $B^{p} \ldots \ldots \ldots 41$

Thomas W. Cusick, Integer multiples of periodic continued fractions . . . . . 47

Robert D. Davis, The factors of the ramification sequence of a class of

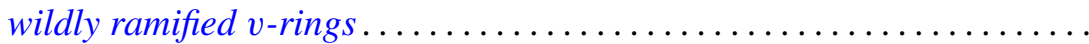

Robert Martin Ephraim, Multiplicative linear functionals of Stein

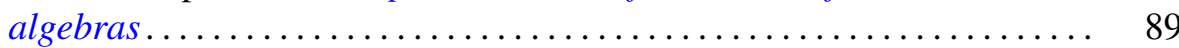

Philip Joel Feinsilver, Operator calculus . .................... 95

David Andrew Gay and William Yslas Vélez, On the degree of the splitting field of an irreducible binomial ..........................

Robert William Gilmer, Jr. and William James Heinzer, On the divisors of

monic polynomials over a commutative ring ..................

Robert E. Hartwig, Schur's theorem and the Drazin inverse .............

Hugh M. Hilden, Embeddings and branched covering spaces for three and four dimensional manifolds ............................

Carlos Moreno, The Petersson inner product and the residue of an Euler product. ...

Christopher Lloyd Morgan, On relations for representations of finite groups....

Ira J. Papick, Finite type extensions and coherence

$\mathrm{R}$. Michael Range, The Carathéodory metric and holomorphic maps on a class of weakly pseudoconvex domains ................

Donald Michael Redmond, Mean value theorems for a class of Dirichlet series

Daniel Reich, Partitioning integers using a finitely generated semigroup ...

Georg Johann Rieger, Remark on a paper of Stux concerning squarefree

numbers in non-linear sequences

Gerhard Rosenberger, Alternierende Produkte in freien Gruppen ..

Ryōtarō Satō, Contraction semigroups in Lebesgue space

Tord Sjödin, Capacities of compact sets in linear subspaces of $\mathbf{R}^{n}$

Robert Jeffrey Zimmer, Uniform subgroups and ergodic actions of exponential Lie groups......................... 